OPEN ACCESS

Edited by:

Diana Boraschi,

Shenzhen Institutes of Advanced Technology, Chinese Academy of

Sciences (CAS), China

Reviewed by:

Laura Rehak,

Athena Biomedical Innovations, Italy

Detlef Neumann,

Hannover Medical School, Germany

*Correspondence:

Xiangwei Xiao

xiangweixiao@chp.edu

Lingyan Zhu

zly982387@126.com

Specialty section:

This article was submitted to

Cytokines and Soluble

Mediators in Immunity,

a section of the journal

Frontiers in Immunology

Received: 04 July 2021 Accepted: 13 September 2021

Published: 29 September 2021

Citation:

Zhu L, Qian J, Jiang Y, Yang T, Duan Q and Xiao X (2021) PIGF Reduction

Compromises Angiogenesis in Diabetic Foot Disease

Through Macrophages.

Front. Immunol. 12:736153. doi: 10.3389/fimmu.2021.736153

\section{PIGF Reduction Compromises Angiogenesis in Diabetic Foot Disease Through Macrophages}

\author{
Lingyan Zhu ${ }^{1,2 *}$, Jieqi Qian ${ }^{3}$, Yinan Jiang ${ }^{3}$, Tianlun Yang ${ }^{4}$, Qiong Duan ${ }^{5}$ \\ and Xiangwei $\mathrm{Xiao}^{3 *}$
}

\begin{abstract}
1 Department of Endocrinology, The First Affiliated Hospital of Nanchang University, Nanchang, China, ${ }^{2}$ Department of Endocrinology, The Peoples Hospital of Yudu County, Ganzhou, China, ${ }^{3}$ Department of Surgery, Children's Hospital of Pittsburgh, School of Medicine, University of Pittsburgh, Pittsburgh, PA, United States, ${ }^{4}$ Department of Cardiology, Xiangya Hospital, Central South University, Changsha, China, ${ }^{5}$ Department of Cardiology, the First Affiliated Hospital of Nanchang University, Nanchang, China
\end{abstract}

Diabetic foot disease (DFD) is a common and serious complication for diabetes and is characterized with impaired angiogenesis. In addition to the well-defined role of vascular endothelial growth factor (VEGF) -A and its defect in the pathogenesis of DFD, another VEGF family member, placental growth factor (PIGF), was also recently found to alter expression pattern in the DFD patients with undetermined mechanisms. This question was thus addressed in the current study. We detected attenuated PIGF upregulation in a mouse DFD model. In addition, the major cell types at the wound to express the unique PIGF receptor, VEGF receptor 1 (VEGFR1), were macrophages and endothelial cells. To assess how PIGF regulates DFD-associated angiogenesis, we injected recombinant PIGF and depleted VEGF1R specifically in macrophages by local injection of an adenoassociated virus (AAV) carrying siRNA for VEGFR1 under a macrophage-specific CD68 promoter. We found that the angiogenesis and recovery of the DFD were both improved by PIGF injection. The PIGF-induced improvement in angiogenesis and the recovery of skin injury were largely attenuated by macrophage-specific depletion of VEGF1R, likely resulting from reduced macrophage number and reduced M2 polarization. Together, our data suggest that reduced PIGF compromises angiogenesis in DFD at least partially through macrophages.

\section{Keywords: diabetic foot disease (DFD), placental growth factor (PIGF), vascular endothelial growth factor receptor 1 (VEGFR1), macrophages, angiogenesis}

\section{INTRODUCTION}

Vascular endothelial growth factor -A (VEGF-A), a potent endothelial cell mitogen, has been shown to play a substantial role in angiogenesis (1). VEGF is a heparin-bonded glycoprotein that has two specific receptors, VEGF receptor 1 (VEGFR1) and VEGF receptor 2 (VEGFR2) (2). Besides VEGFA, VEGF family has 5 other members, among which placental growth factor (PlGF) is a critical one and has been shown to be associated with pathological angiogenesis (3). 
Peripheral neuropathy and peripheral vascular disorders hallmark the pathology of DFD (4). The past studies have demonstrated defect in VEGF-A-mediated angiogenesis in DFD (5). Our lab has studied diabetes-associated angiogenesis for years (6-8). We have also shown that enhanced VEGF-A translation through genetic modulation of non-coding RNAs in human mesenchymal stem cells (MSCs) promotes the secretion of VEGF-A by MSCs to improve the angiogenesis and recovery of the injury at the wound site in DFD $(9,10)$. Recently, we have shown that PlGF plays a critical role in gestational beta-cell growth (11), likely as a potent growth factor that regulates the crosstalk between trophic macrophages and pancreatic beta-cells (11). Interestingly, upregulation of PlGF has recently been shown during wound healing, and the levels of PlGF were reduced in DFD patients (12), and in mouse models for DFD (13). In another study that investigated the role of PlGF in diabetic wound healing, the effects of PlGF on fibroblasts but not on macrophages were investigated (14). Since the major cell types that harbor the unique PlGF receptor VEGFR1 are macrophages, endothelial cells and fibroblasts (2), the exact molecular mechanisms underlying PlGF-regulated angiogenesis in DFD seems warrant further investigation, which was thus addressed in the current study.

We detected attenuated PlGF upregulation in a mouse DFD model. In addition, the major cell types at the wound to express the unique PlGF receptor, VEGFR1, were macrophages and endothelial cells. To assess how PlGF regulates DFD-associated angiogenesis, we injected recombinant PlGF and depleted VEGF1R specifically in macrophages by local injection of an adeno-associated virus (AAV) carrying siRNA for VEGFR1 under a macrophage-specific CD68 promoter. We found that the angiogenesis and the recovery of the DFD were both improved by PlGF injection but were largely attenuated by macrophage-specific depletion of VEGF1R, likely resulting from reduced macrophage recruitment and reduced M2 polarization.

\section{MATERIALS AND METHODS}

\section{Protocol Approval}

All the experimental protocols, animal surgery and treatments applied in the current study have been approved by the research committee and Institutional Animal Care and Use Committee at the First Affiliated Hospital of Nanchang University, respectively. The experiments were carried out in accordance with the approved guidelines. For each animal experiment, a power calculation $(p<0.05)$ was performed to determine exactly sufficient number of mice to allow the observed effects to be legitimate. An allocation concealment method was used to provide randomization in allocating experimental units to control and treatment groups. The use of the inbred littermate mice in a specified experiment ensured that the potential confounders were minimized. No criteria were used for excluding animals (or experimental units) during the experiment, and no data were excluded during the analysis.

\section{Induction of Diabetes, DFD and AAV Transplantation}

Randomization and blind assessment were used in all animal studies. Diabetes was induced in 12-week-old C57/BL6 mice (male and female are evenly distributed in each group, SLAC Laboratory Animal, Shanghai, China) by single intraperitoneal injection of $160 \mathrm{mg} / \mathrm{kg}$ streptozotocin (STZ) in $100 \mu \mathrm{l}$ normal saline after a 12-hour fasting, as described before (15). This dose used in fasting wildtype mice results in 100\% development of diabetes, since STZ specially goes into beta-cells through glucose transporter 2 (Glut2) on beta-cells. Fasting is critical since circulating glucose can compete with STZ for Glut2 to compromise the effects of STZ. The control mice received equal volume of the normal saline, the solvent to dissolve STZ. One week later, all mice that had received STZ became hyperglycemic (fasting blood glucose $>=350 \mathrm{mg} / \mathrm{dl}$ ). If any mice did not develop hyperglycemia (fasting blood glucose $>=350 \mathrm{mg} / \mathrm{dl}$ ) one week after STZ, they were supposed to be excluded from this study. The STZ-treated mice then underwent a surgery to create a $6 \mathrm{~mm}$ diameter full-thickness wound on the dorsal midline with a biopsy punch, with/without orthotopic injection with $100 \mu \mathrm{l}$ AAV viruses $\left[10^{11}\right.$ genome copy particle $\left.(\mathrm{GCP}) / \mathrm{ml}\right]$, as described (16). Meanwhile, the wounds of the experimental mice were treated with $50 \mu \mathrm{l}$ recombinant PlGF $(10 \mu \mathrm{g}$, Ab207150, Abcam, Cambridge, MA, USA) or an equivalent volume of saline solution a Hamilton's syringe under the clot covering the wound at a frequency of twice per week. Fasting blood glucose were performed at 10am after a three-hour fasting period and assessment of beta-cell mass was done as described $(17,18)$. For vessel density measurement, tomato-lectin $(50 \mu \mathrm{l}$, Vectorlabs, Burlingame, CA USA) was injected to the mice through the tail vein 10 minutes before sacrifice. The vessel density was determined bases on the percentage of lectionpositive area in total measured tissue area, as described before (10).

\section{Production of AAVs}

Transfection of human embryonic kidney 293 cells by AAV serotype 6 vectors were applied by as described before $(19,20)$. The backbone plasmid and the plasmid containing human CD68 promoter were both obtained from an Addgene plasmid (\#32395 (21) and \#34837 (22), Watertown, MA, USA). The sequence for siRNA for VEGFR1 was 5'-CCAGACACTGCATCCAA-3', and the control scramble sequence was 5'-GCATTAACTA AAGGCUGCC-3'. The Transfection was performed with Lipofectamine 3000 reagent (Invitrogen, CA, Carlsbad, USA). Purification and titration of AAV vectors were performed with standard procedure and a dot-blot assay, respectively, as described before (19).

\section{Flow Cytometry}

The injured skin tissue was dissected out and digested with $0.25 \%$ trypsin for 35 minutes to obtain a single cell fraction for flow cytometry. The flow cytometry-based cell purification was based on direct fluorescence from GFP (AAVs-originated) and Tomato (Lectin-originated) as well as immunofluorescence from 
VEGFR1 (by a PE-cy7-conjugated anti-VEGFR1 antibody, Becton-Dickinson Biosciences, San Jose, CA, USA) or CD68 (by a BV421-conjugated anti-CD68 antibody) or CD31 (by an APC-conjugated anti-CD68 antibody), as described before (15). The flow cytometry data were analyzed by Flowjo (version 11.0, Flowjo LLC, Ashland, OR, USA).

\section{Quantitative Real-Time PCR (RT-qPCR)}

A RNeasy mini kit (Qiagen, Germantown, MD, USA) was used to extract RNA. Quantitative real-time PCR (RT-qPCR) were performed using commercial primers ordered from Qiagen. Data were collected and analyzed using 2- $\triangle \triangle \mathrm{Ct}$ method. Values of genes were obtained by sequential normalization against the internal control GAPDH and the corresponding experimental controls.

\section{ELISA}

The extracted protein by RIPA buffer was subjected to mouse PlGF-2 (MP200) and VEGFR1 (MVR100) enzyme-linked immunosorbent assay (ELISA; R\&D System, Los Angeles, CA, USA), as described before (10). The microplate was read at 450 $\mathrm{nm}$ within 30 minutes. The wavelength correction was set to 540 $\mathrm{nm}$ or $570 \mathrm{~nm}$.

\section{Histology and Immunostaining}

Ten minutes after tomato-lectin infusion through the tail vein, the mice were sacrificed. The pancreas and the injured skin were dissected out and fixed in 4\% paraformaldehyde (PFA, SigmaAldrich, St. Louis, MO, USA) for 6 hours. After incubation in $30 \%$ sucrose for 24 hours, the samples were frozen and embedded. Fluorescent immunostaining was done as described before (10). Tomato-lectin was detected by direct red fluorescence and virus-transduced macrophages were detected by GFP. Immunofluorescent staining for glucagon and insulin was performed with a monoclonal antibody against glucagon (Ab10988, Abcam) and a guinea pig polyclonal antibody against insulin (Ab7842, Abcam), respectively. Hoechst 33342 (HO, Sigma-Aldrich) was applied to stain the nucleus.

\section{Bioinformatics}

Gene Expression Omnibus (GEO, http://www.ncbi.nlm.nih.gov/ geo/) was used to obtain data for bioinformatic analysis (23). The data surf applied "diabetic foot" OR "diabetic foot disease "AND "Homo sapiens". After a careful assessment for relatedness, two gene expression profiles (GSE80178 and GSE134431) were selected (24). The GEO2R online analysis tool and "DESeq2" R package were applied for determining the differentially expressed genes (DEGs) and calculation for P-value, adjusted P-value and $\operatorname{logFC}$. PlGF levels in both series were obtained and analyzed the difference among groups. Pathway enrichment analyses of DEGs were performed at Metascape (http://metascape.org).

\section{Statistical Analysis}

Individual values were represented in the figures. Statistical analysis was carried out using a one-way analysis of variance (ANOVA) test followed by the Fisher's Exact Test (GraphPad
Software, version 9, Inc. La Jolla, CA, USA). A value of $\mathrm{p}<0.05$ was considered statistically significant.

\section{RESULTS}

\section{PIGF Upregulates During Foot Wound Healing and This Upregulation Is Compromised in Diabetes}

Since recent studies revealed a possible role of PlGF in DFD, we analyzed tissue PlGF levels at day 3 and day 7 after induction of DFD in mice $(9,10)$. PlGF levels in diabetic foot skin (or diabetic ulcer (DU) at day 0), did not differ from non-diabetic controls (or non-diabetic ulcer (NDU) at day 0) (Figure 1A). PlGF levels increased at both day 3 and day 7 after ulcer induction, regardless of the diabetic status (Figure 1A), which was consistent with the analysis on 2 human DFD datasets (GSE134431, Figure 1B; GSE80178, Figure 1C). However, the overall increases in PlGF levels were significantly attenuated in diabetes (Figure 1A). The analysis on human DFD dataset GSE80178 also showed significant difference in gene expression (Figure 1D) and vasculature development pathway (Figure 1E). Together, these data highlight the importance of angiogenesis and suggest a possible role of PlGF in the pathological process of DFD.

\section{VEGFR1 Is Expressed in Macrophages, Endothelial Cells and Fibroblasts in DFD Tissue}

Next, we investigated the role of PlGF in angiogenesis and the wound recovery in DFD. Since PlGF has a unique receptor, VEGFR1, we analyzed the cell types that harbor VEGFR1 to respond to PlGF at the wound. In a previous publication, macrophages, endothelial cells and fibroblasts have been shown to express VEGFR1 (2). To confirm it, day 3 injured skin tissue was digested into single cell population and then sorted for VEGFR1+ cells versus VEGFR1- cells by flow cytometry (Figure 2A). Afterwards, the VEGFR1+ cells were further sorted for CD68 (a marker for macrophages) and CD31 (a marker for CD31). Therefore, the VEGFR1+ cells were in addition separated as VEGFR1+CD68+ cells (macrophages), VEGFR1+CD31+ cells (endothelial cells), and VEGFR1+CD68-CD31- cells (VEGFR1+ cells that are not macrophages or endothelial cells) (Figure 2A). We found that among all VEGFR1+ cells, either CD68+ cells or CD31+ cells are much more than CD68-CD31- cells, suggesting that most VEGFR1+ cells are either macrophages or endothelial cells (Figure 2A). RT-qPCR was then performed on the sorted cells, showing significantly higher VEGFR1 in all 3 VEGFR1+ populations than VEGFR1- population, significantly higher levels of CD68 in VEGFR1+CD68+ cell population than the other 3 cell populations, significantly higher levels of CD31 in VEGFR $1+C D 31+$ cell population than the other 3 cell populations, and significantly higher levels of Vimentin (a marker for fibroblasts) in VEGFR1+CD68-CD31- cell population than the other 3 cell populations (Figure 2B), with the latter suggests that most fibroblasts are in the VEGFR1+CD68- 
A

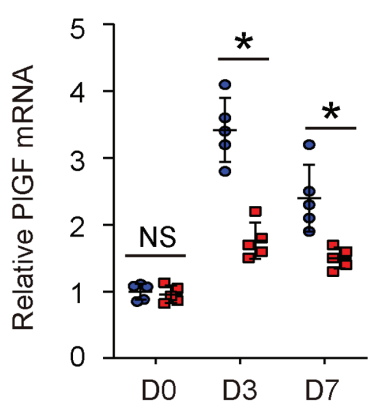

B

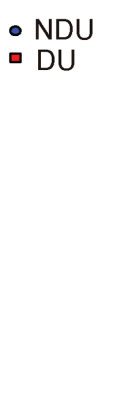

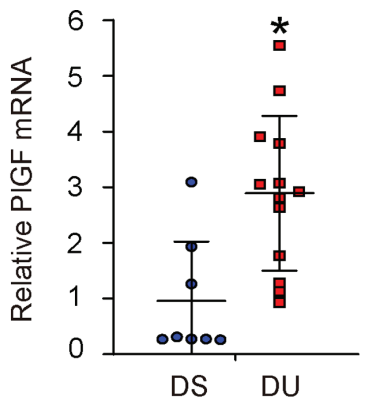

C

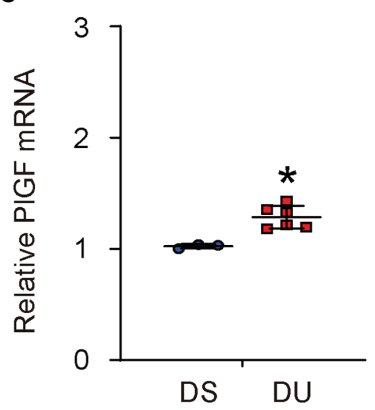

D

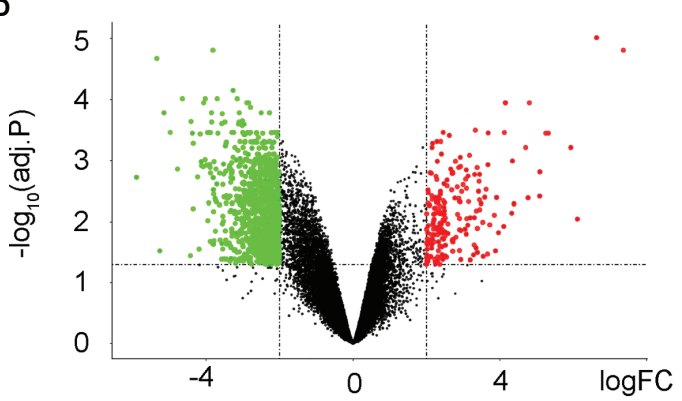

E

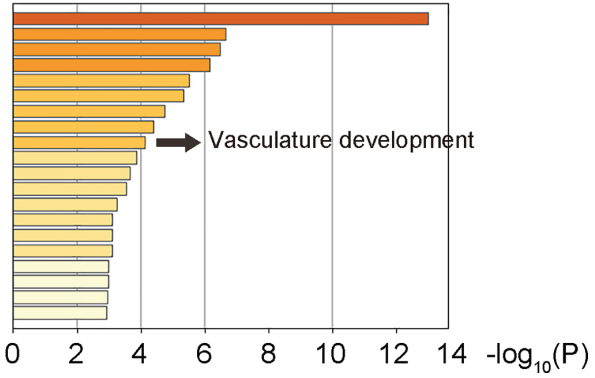

FIGURE 1 | PIGF upregulates during foot wound healing and this upregulation is compromised in diabetes. (A) ELISA for PIGF in diseased foot tissue from a mouse model for DFD before induction of ulcer (day 0) and day 3 and day 7 after induction of DFD in mice. DU: diabetic ulcer. NDU: non-diabetic ulcer. (B, C) PIGF mRNA levels in diabetic foot skin (DS, equal to DU day 0) versus diabetic foot ulcer (NDU, equal to DU day 3) from human DFD dataset GSE134431 (B) and GSE80178 (C) (D) A hot map to show difference in gene expression from DS versus DU in GSE80178. (E) A pathway analysis to show difference in enriched pathway between DS and DU in GSE80178. * $p<0.05$. NS, non-significant.

A

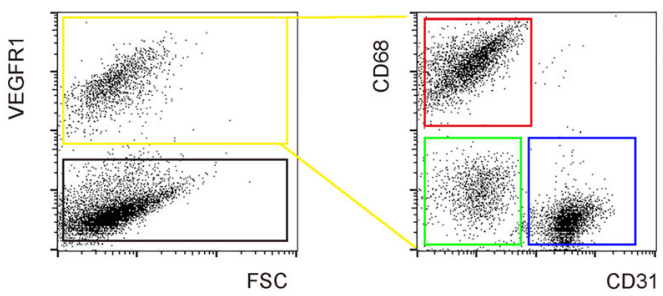

B

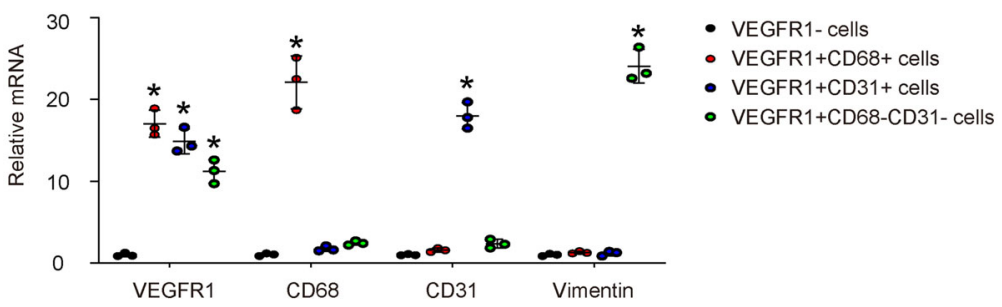

FIGURE 2 | VEGFR1 is expressed in macrophages, endothelial cells and fibroblasts in DFD tissue. (A) A representative flow chart to show digestion of day 3 DFD tissue into single cell population and a subsequent sorting of VEGFR1+ cells versus VEGFR1- cells by flow cytometry. Afterwards, the VEGFR1+ cells were sorted for CD68 and CD31 to further separate VEGFR1+ cells into VEGFR1+CD68+ cells (macrophages), VEGFR1+CD31+ cells (endothelial cells), and VEGFR1+CD68-CD31cells (VEGFR1+ cells that are not macrophages or endothelial cells). (B) RT-qPCR for VEGFR1, CD68, CD31 and vimentin on the sorted 4 cell fractions. ${ }^{*} p<0.05$. 
CD31- cell population. Compared with results from Figure 2A, fibroblasts may represent a relatively smaller cell population that expresses VEGFR1.

\section{Generation of AAVs That Deplete VEGFR1 Specifically in Macrophages}

The effects of PlGF on fibroblasts but not on macrophages during tissue repair have been investigated (14). Since macrophages are known to play a critical role in angiogenesis, and since we detected a great number of macrophages among all VEGFR1+ cells in the mouse DFD model (Figure 2A), here we focused on macrophages. We generated an AAV carrying siRNA for VEGFR1 under a macrophage-specific CD68 promoter (AAVpCD68-siVEGFR1), which allows specific depletion of VEGFR1 in macrophages and allows us to assess the effects of PlGF on angiogenesis and recovery of the DFD injury through macrophages (Figure 3A). To ensure the specificity of this construct, isolated macrophages, endothelial cells and mouse embryonic fibroblasts (MEFs), 3 cell types that express VEGFR1, were infected with either AAV-pCD68-siVEGFR1 or control AAV-pCD68-SCR. We found that CD68 promoter allowed effective transduction of macrophages, but not endothelial cells or MEFs, based on the expression of the GFP reporter
(Figure 3B). The levels of VEGFR1 in the infected cells were analyzed by RT-qPCR, showing significant reduction in VEGFR1 mRNA in AAV-pCD68-siVEGFR1-transduced macrophages, while the VEGFR1 mRNA in either endothelial cells or MEFs remained unchanged (Figure 3C). Similar results were obtained when VEGFR1 protein was analyzed by ELISA (Figure 3D). Thus, AAV-pCD68-siVEGFR1 specifically depletes VEGFR1 in macrophages.

\section{PIGF With/Without Macrophage-Depletion of VEGFR1 Does Not Affect Diabetes}

The effects of PIGF on DFD and the role of macrophages were then assessed in a mouse model for DFD, in which C56/BL6 mice received STZ to induce diabetes and then received a surgical ulcer at dorsal midline after one week. At the time of surgical ulcer induction, PlGF or saline was orthotopically injected biweekly while AAVs were given locally to the wound only once, immediately after ulcer induction. We have previously shown that the AAV-mediated transduction in vivo can sustain much longer than the one month's experimental time window (25-27). After another 4 weeks, the mice were sacrificed and analyzed (Figure 4A). We found that STZ treatment induced irreversible hyperglycemia in mice, while any treatments or
A

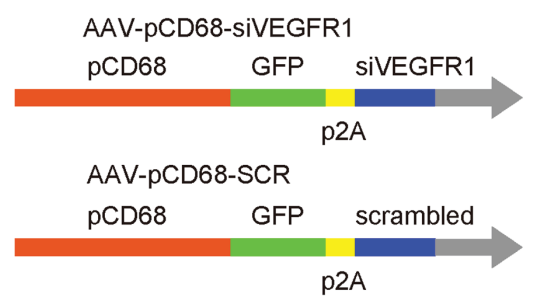

C

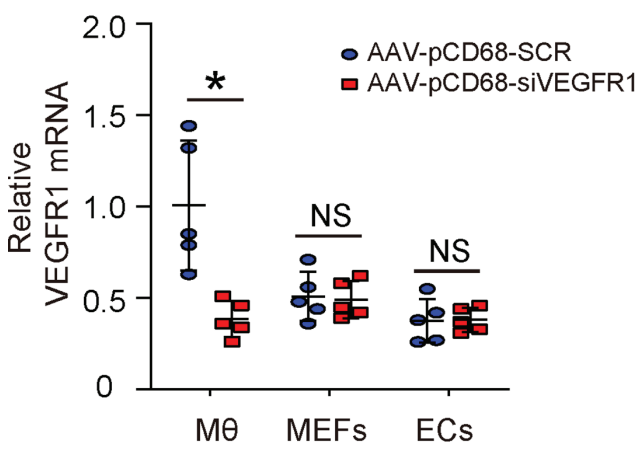

B

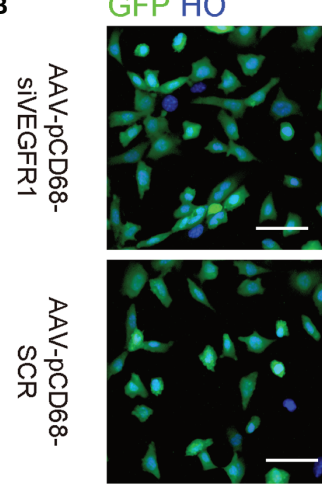

$\mathrm{M} \theta$

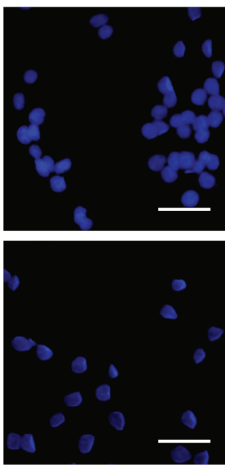

MEFs

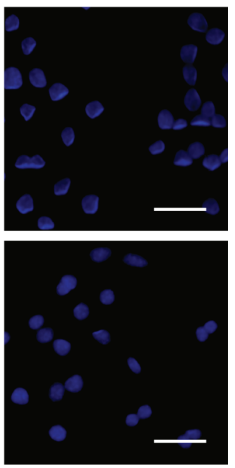

ECs
D

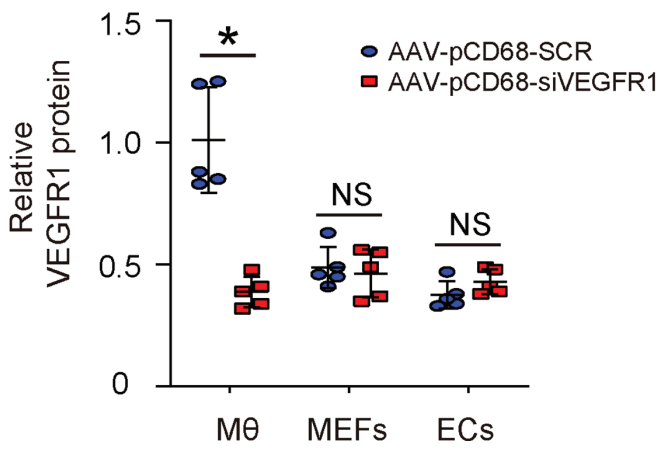

FIGURE 3 | Generation of AAVs that deplete VEGFR1 specifically in macrophages. (A) Schematic of an AAV carrying siRNA for VEGFR1 under a macrophagespecific CD68 promoter (AAV-pCD68-siVEGFR1) and control AAV-pCD68-SCR. (B) Isolated macrophages (M9), endothelial cells (ECs) and mouse embryonic fibroblasts (MEFs) were infected with AAV-pCD68-siVEGFR1 or control AAV-pCD68-SCR, shown by representative immunofluorescent images. (C, D) RT-qPCR (C) and ELISA (D) for VEGFR1 in infected Me, ECs and MEFs. ${ }^{*} \mathrm{p}<0.05$. NS, non-significant. Scale bars are $20 \mu \mathrm{m}$. 
A

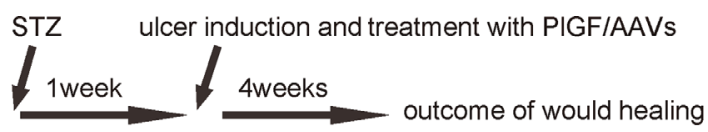

B

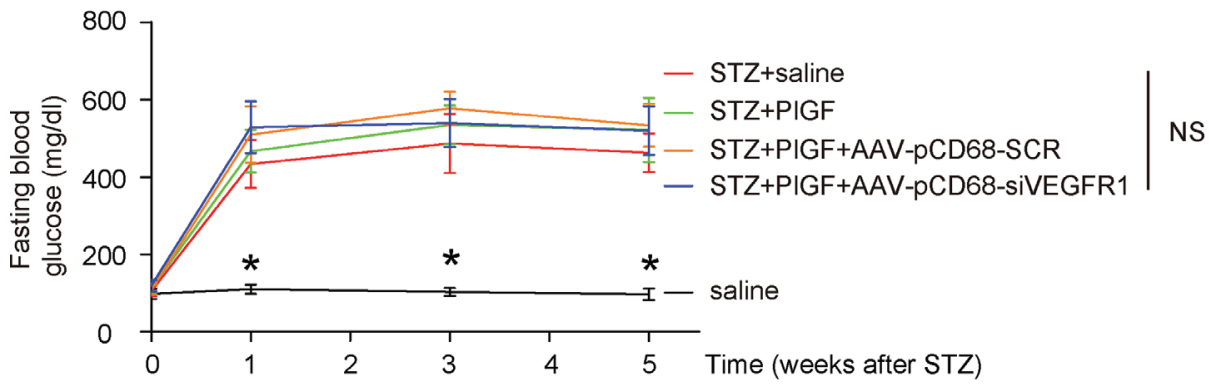

C

insulin glucagon $\mathrm{HO}$

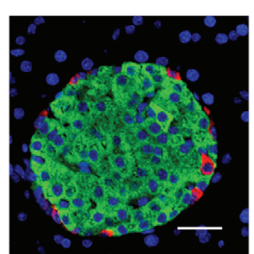

saline

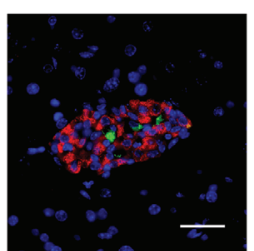

STZ+saline

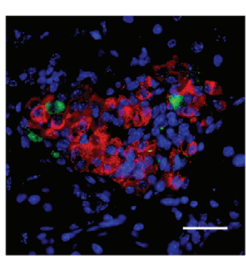

STZ+PIGF

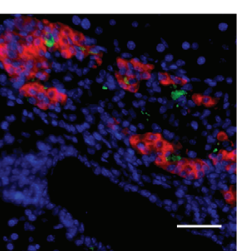

STZ+PIGF+ AAV-pCD68-SCR AAV-pCD68-siVEGFR1

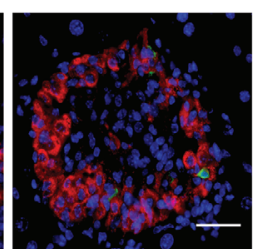

$\mathrm{STZ}+\mathrm{PIGF}+$

D

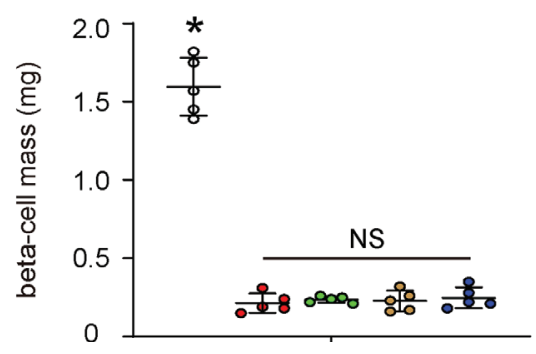

- saline

- STZ+saline

- $S T Z+P I G F$

- $S T Z+P I G F+A A V-p C D 68-S C R$

- STZ+PIGF+AAV-pCD68-siVEGFR1

FIGURE 4 | PIGF with/without macrophage-depletion of VEGFR1 does not affect diabetes. (A) Schematic of a mouse model for DFD, in which C56/BL6 mice received STZ to induce diabetes and then a surgical ulcer at dorsal midline after one week. At the time of surgical ulcer induction, PIGF or saline was orthotopically injected biweekly while AAVs were given locally only once, immediately after ulcer induction. In another 4 weeks, the mice were sacrificed and analyzed. (B) Fasting blood glucose. (C) Representative immunofluorescent images for glucagon and insulin in mouse pancreas. (D) Beta-cell mass at sacrifice. ${ }^{*} p<0.05$. NS, nonsignificant. Scale bars are $20 \mu \mathrm{m}$.

treatment combinations neither correct hyperglycemia (Figure 4B), nor alter beta-cell mass (Figures 4C, D). These data suggest that the sustained hyperglycemia induced by STZ resulted from loss of beta-cells rather than from other reasons, i.e. insulin resistant, in which hyperglycemia is developed while the beta cell number can remain normal or even increase at the early stage (28). Moreover, PlGF treatment with/without macrophage-depletion of VEGFR1 does not affect diabetes.

\section{PIGF Promotes Wound Healing and Angiogenesis, Which Are Attenuated by Macrophage-Depletion of VEGFR1}

Next, we examined the effects of PlGF through macrophages on the wound healing in DFD. We found that the wound was completely cured at 4 weeks after ulcer induction in salinetreated normoglycemic mice (saline) (Figure 5A). The ulcers did not cure in all STZ-treated mice. However, better recovery was detected in diabetic mice treated with PlGF (Figure 5A). This improvement of wound recovery by PlGF, was however, abolished by macrophage-depletion of VEGFR1 (Figure 5A). Angiogenesis was analyzed at the end of the experiment by vessel density ( 5 weeks after STZ or 4 weeks after PlGF/AAVs). We found that the vessel density was significantly reduced by STZ treatment in the injured tissue (Figures 5B, C). However, improved angiogenesis was detected in diabetic STZ-mice treated with PlGF (Figures 5B, C). This improved angiogenesis by PIGF was also abolished by macrophage-depletion of VEGFR1 (Figures 5B, C). Thus, PlGF promotes wound 
A

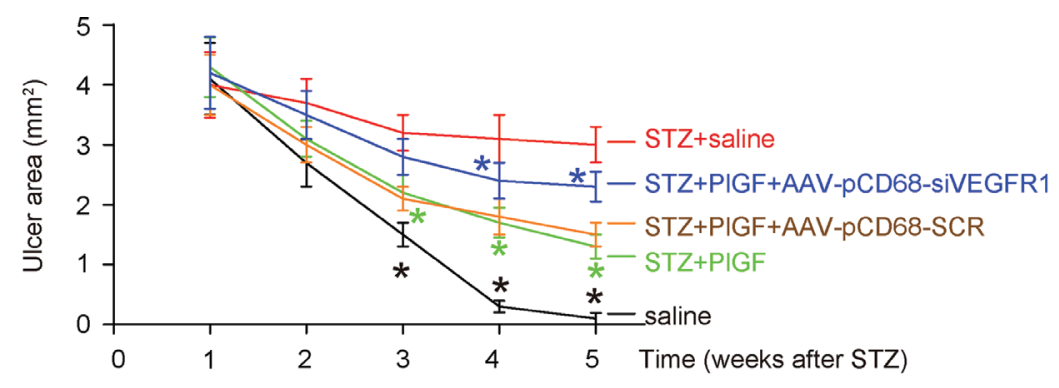

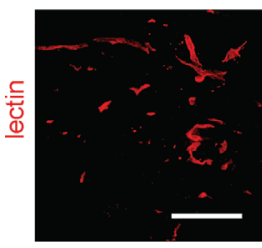

saline

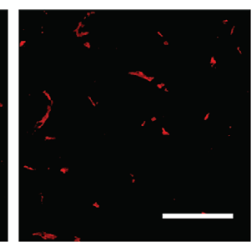

STZ+saline

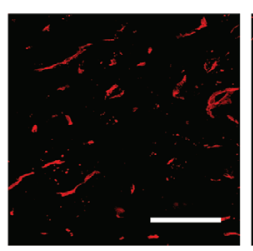

STZ+PIGF

C

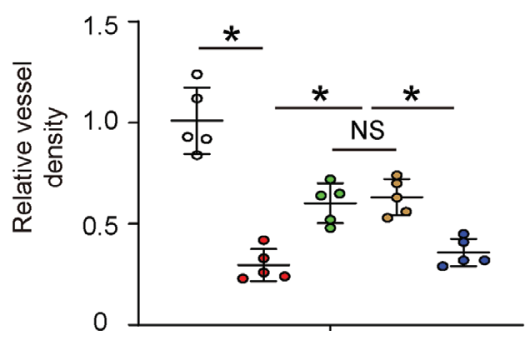

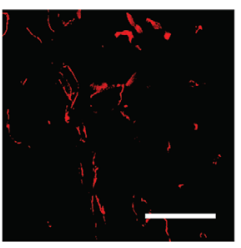

STZ+PIGF+

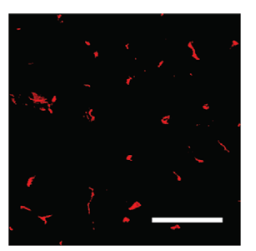

STZ+PIGF+

- saline

- STZ+saline

- $\mathrm{STZ}+\mathrm{PIGF}$

- STZ+PIGF+AAV-pCD68-SCR

- STZ+PIGF+AAV-pCD68-siVEGFR1

FIGURE 5 | PIGF promotes wound healing and angiogenesis, which are attenuated by macrophage-depletion of VEGFR1. (A) The effects of PIGF through macrophages on wound healing of DFD were examined. (B, C) Angiogenesis was analyzed at the end of the experiment by vessel density (5 weeks after STZ or 4 weeks after PIGF/ AAVs), shown by representative fluorescent images (B) and by quantification (C). ${ }^{*} \mathrm{p}<0.05$. In panel (A), black star: STZ vs saline, green star: STZ+PIGF vs STZ, blue star: STZ+PIGF+AAV-pCD68-siVEGFR1 vs STZ+PIGF+AAV-pCD68-SCR. NS, non-significant. Scale bars are $100 \mu \mathrm{m}$.

healing and angiogenesis, which are likely through VEGFR1 on macrophages.

\section{Macrophage-Depletion of VEGF1R Reduces the Number of Macrophages and Reduces M2 Polarization}

Since we found that PlGF-induced improvement in angiogenesis and recovery of DFD was largely attenuated by macrophagespecific depletion of VEGF1R, we examined the changes in macrophages in response to PlGF and VEGFR1 depletion. First, PlGF in tissue was assessed in all experimental groups, showing significant reduction by STZ and significant increase by PlGF (Figure 6A). Surprisingly, PlGF levels were reduced by macrophage-depletion of VEGFR1 (Figure 6A). Next, macrophages (CD68+) and their M2 (CD68+CD163+) versus M1 (CD68+CD163-) polarization were analyzed by flow cytometry (Figures 6B-D). Interestingly, we found that STZ not only reduced the number of macrophages, but also reduced their M2 polarization (Figures 6B-D). Moreover, PlGF not only increased the number of macrophages, but also enhanced their M2 polarization (Figures 6B-D). Finally, the effects of PlGF on both macrophage number and their M2 polarization were both attenuated by macrophage-depletion of VEGFR1 (Figures 6B-D).

\section{DISCUSSION}

Wound healing is orchestrated by a number of biological events that include tissue hemostasis, local inflammation, cell proliferation, and structure remodeling, during which both macrophages and vascular endothelial cells play a pivotal role (29). Endothelial cells are activated by hypoxia and increase vascular permeability to recruit inflammatory cells including macrophages to the wound site (29). The interaction between endothelial cells and macrophages alters the phenotypes of each other to allow not only the oxygen and nutrient transit from circulation to promote cell proliferation and differentiation, but also the production and release of growth factors like proangiogenic factors from macrophages to affect the formation of new capillaries through angiogenesis (30). Among these factors, the most potent one is VEGF-A, which has been known as the most important angiogenic factor in wounds (31). However, 
A

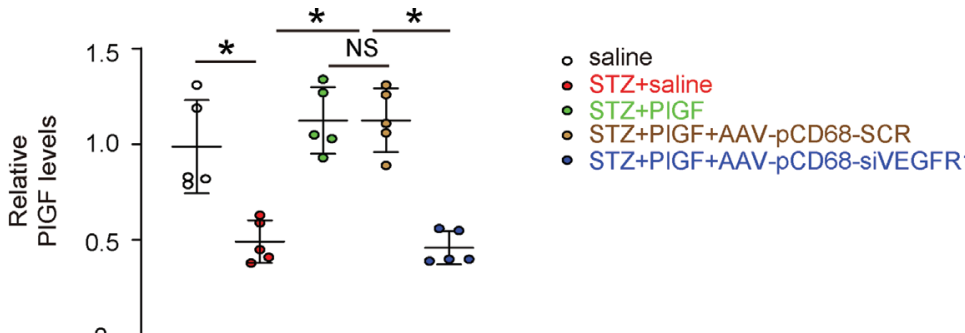

B
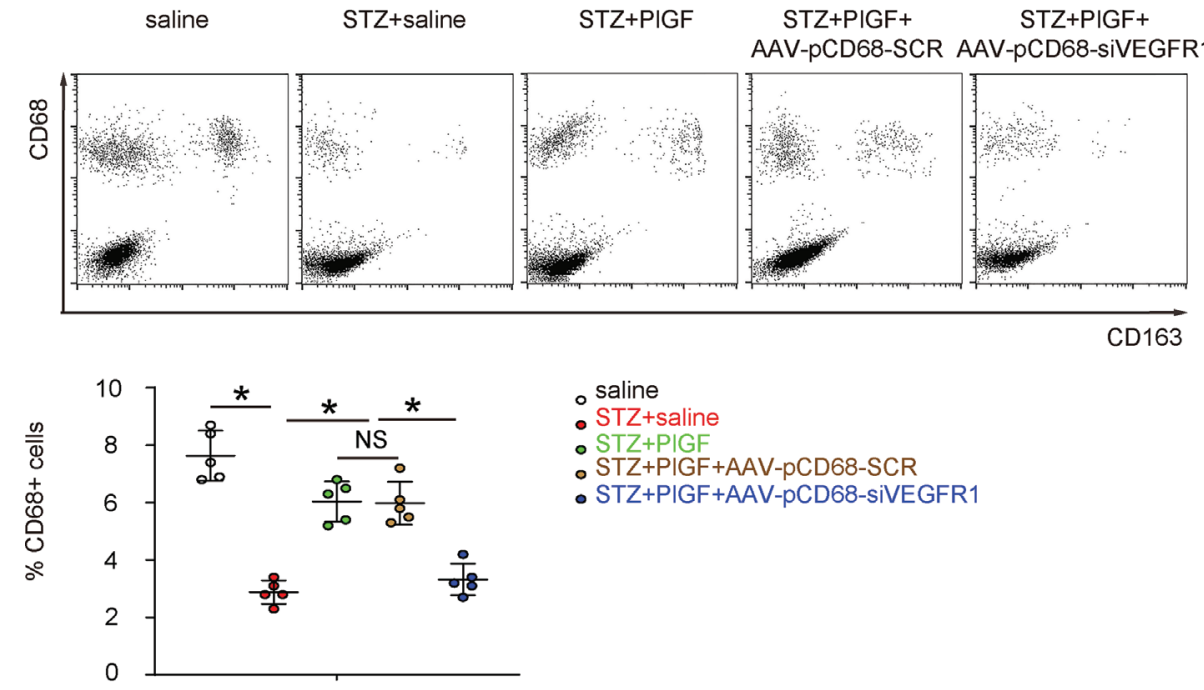

D

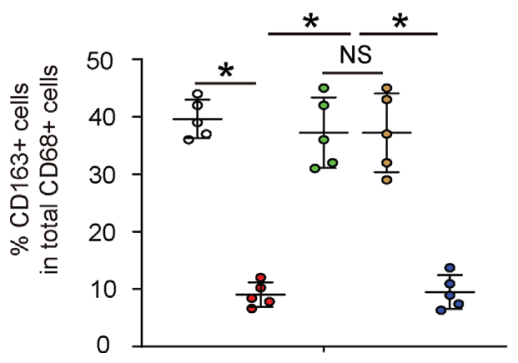

- saline

- STZ+saline

- $\mathrm{STZ}+\mathrm{PIGF}$

- STZ+PIGF+AAV-pCD68-SCR

- STZ+PIGF+AAV-pCD68-siVEGFR1

FIGURE 6 | Macrophage-depletion of VEGF1R reduces macrophage number and reduces M2 polarization. (A) ELISA for PIGF levels in tissue from all experimental groups. (B) Representative flow charts for analysis and sorting for CD68 and CD163. (C, D) Quantification of CD68+ macrophage percentage (C) and CD163+ cells in total CD68+ cells (D). * $p<0.05$. NS, non-significant.

recent studies have demonstrated a previously neglected role of PlGF in angiogenesis, especially in diabetes (14), as it is likely more important in pathological angiogenesis (32).

Here, we showed reduced PlGF in DFD, and correction of PlGF could improve the impaired angiogenesis and wound recovery of DFD, suggesting that PlGF, in addition to VEGF$A$, may play a substantial role in angiogenesis under a diabetic status. Unlikely VEGF-A, which has two receptors VEGFR1 and VEGFR2, PlGF functions exclusively through VEGFR1 (33). Interestingly, both VEGFR1 and VEGFR2 are expressed by endothelial cells, while previous studies have shown that VEGFR1 on endothelial cells appears to have a higher affinity but a lower potency with VEGF-A, compared to VEGFR2 (34). Thus, the PlGF/VEGFR1 signaling may play a negative role in endothelial cells during angiogenesis. That is the reason that we did not investigate the effect of PlGF on endothelial cells in the current study. On the other hand, PlGF/VEGFR1 signaling is critical for macrophages that harbor VEGFR1 but not VEGFR2 (35). Specially, PlGF has been shown to induce polarization of macrophage to a M2-like subtype, which is important for cell proliferation, regeneration and tissue remodeling, as we showed before (27). This concept has been well confirmed in the current study, as we detected potential increases in the number of macrophages and their M2 polarization induced by PlGF. Macrophages that have undergone a M2 polarization may release PlGF to bind VEGFR1 on their own to create an autocrine positive regulatory loop to amplify the effect, as reported before (36), which could explain why CD68+ cell 
number and CD163+ M2 macrophage percentage in total macrophages were both reduced by macrophage-depletion of VEGFR1 (Figures 6B-D). Indeed, since VEGFR1 is the only receptor for PlGF, PlGF secreted by injured tissue or by the experimental administration (recombinant PlGF injeciton) can recruit macrophages via their VEGFR1, which is a typical chemokine effect. On the other hand, siRNA for VEGFR1 under the control of a macrophage-specific CD68 promoter allows exclusive transduction of macrophages by siVEGFR1, resulting in the loss of the response of macrophages to PlGF. Hence, the PlGF/VEGFR1 regulatory axis is abolished to prevent a positive effect on the macrophage polarization and migration, during which macrophages are induced to produce and secrete PlGF to create an autocrine effect to amplify this effect (36). Given the relative lower effect of fibrosis on angiogenesis and the unique negative effect of PlGF on angiogenesis through VEGFR1 on endothelial cells, the importance of PlGF to the diabetic angiogenesis may largely result from its effect on macrophages, as shown in this study.

The finding in this study is well in line with some clinical trials of autologous cell therapy based on peripheral blood mononuclear cells implant (PBMNC) in critical limb ischemia patients. First, autologous PBMNC implant is shown to increase angiogenesis and wound healing in critical limb-ischemic diabetic patients and to reduce amputations (37). Second, PBMNC, but not bone marrow mononuclear cells or bone marrow mesenchymal cells, has been found to reduce limb amputation in critical limb-ischemic diabetic patients (38). Third, the autologous PBMNC implants in unhealing wound increase both the macrophage number, VEGF paracrine release and M2 polarization of macrophages (39). Moreover, a recent paper showed that some biomaterials increase and accelerate healing in diabetic foot inducing M2 polarization (40).

The trophic factors produced and released by M2-polarized macrophages, were however, not determined in the current study. In future, RNA-seq analysis could be performed to figure out the growth factors important to macrophageenhanced angiogenesis. Another limitation of the current study is the use of the rodent model, which only partially mimics the DFD in humans. However, it provides important information regarding the underlying molecular mechanisms that can be translatable to human research. Future approaches may assess the role of PlGF and macrophages in non-human primates, which could help to extrapolate the findings in the current study to the therapy in human patients.

\section{REFERENCES}

1. Carmeliet P, Jain RK. Molecular Mechanisms and Clinical Applications of Angiogenesis. Nature (2011) 473:298-307. doi: 10.1038/nature10144

2. Stuttfeld E, Ballmer-Hofer K. Structure and Function of VEGF Receptors. IUBMB Life (2009) 61:915-22. doi: 10.1002/iub.234

3. Eichmann A, Simons M. VEGF Signaling Inside Vascular Endothelial Cells and Beyond. Curr Opin Cell Biol (2012) 24:188-93. doi: 10.1016/j.ceb.2012.02.002

4. Falanga V. Wound Healing and Its Impairment in the Diabetic Foot. Lancet (2005) 366:1736-43. doi: 10.1016/S0140-6736(05)67700-8
To summarize, here we used specific gain-of-function and loss-of-function experiments to demonstrate a role of PlGF in DFD, which is largely mediated by macrophages and their polarization. Our study should provide strong evidence for investigating DFD through novel therapeutic targets.

\section{DATA AVAILABILITY STATEMENT}

The original contributions presented in the study are included in the article/supplementary material. Further inquiries can be directed to the corresponding authors.

\section{ETHICS STATEMENT}

The animal study was reviewed and approved by the First Affiliated Hospital of Nanchang University.

\section{AUTHOR CONTRIBUTIONS}

LZ, JQ, YJ, TY, DD and XX are responsible for data acquisition and analysis. JQ and XX performed bioinformatics analysis. LZ and $\mathrm{XX}$ are responsible for study conception and design, data acquisition and analysis. XX wrote the manuscript and all authors have read the manuscript and agreed with the publication. LZ and XX are responsible for funding and are the guarantee of the study. All authors contributed to the article and approved the submitted version.

\section{FUNDING}

This work was supported by grants from an Assistant Professor Startup from University of Pittsburgh (To XX), National Natural Science Foundation of China (NO 82160155 and 81860153 to LZ, NO 81770358 to TY, China Traditional Chinese and Western Medicine Committee Hehuang Scientific Research Fund (NO 2019005 to LZ), Key Project of Natural Science Foundation of Jiangxi Province (NO 20202ACBL216007 to LZ), Education and Teaching Program of Nanchang University (NO NCUYJSJG-2021-068 and 20190054 to LZ) and Science and Technology Plan of Health Commission of Jiangxi Province (No 20203106 to LZ).

5. Brem H, Tomic-Canic M. Cellular and Molecular Basis of Wound Healing in Diabetes. J Clin Invest (2007) 117:1219-22. doi: 10.1172/JCI32169

6. Zhu L, Xu J, Liu Y, Gong T, Liu J, Huang Q, et al. Prion Protein Is Essential for Diabetic Retinopathy-Associated Neovascularization. Angiogenesis (2018) 21:767-75. doi: 10.1007/s10456-018-9619-4

7. Xiao X, Prasadan K, Guo P, El-Gohary Y, Fischbach S, Wiersch J, et al. Pancreatic Duct Cells as a Source of VEGF in Mice. Diabetologia (2014) 57:991-1000. doi: 10.1007/s00125-014-3179-y

8. Xiao X, Guo P, Chen Z, El-Gohary Y, Wiersch J, Gaffar I, et al. Hypoglycemia Reduces Vascular Endothelial Growth Factor a Production by Pancreatic Beta 
Cells as a Regulator of Beta Cell Mass. J Biol Chem (2013) 288:8636-46. doi: 10.1074/jbc.M112.422949

9. Zhu L, Zhong Q, Yang T, Xiao X. Improved Therapeutic Effects on Diabetic Foot by Human Mesenchymal Stem Cells Expressing MALAT1 as a Sponge for microRNA-205-5p. Aging (2019) 11:12236-45. doi: 10.18632/ aging. 102562

10. Zhu L, Wang G, Fischbach S, Xiao X. Suppression of microRNA-205-5p in Human Mesenchymal Stem Cells Improves Their Therapeutic Potential in Treating Diabetic Foot Disease. Oncotarget (2017) 8:52294-303. doi: 10.18632/oncotarget.17012

11. Yang W, Jiang Y, Wang Y, Zhang T, Liu Q, Wang C, et al. Placental Growth Factor in Beta Cells Plays an Essential Role in Gestational Beta-Cell Growth. BMJ Open Diabetes Res Care (2020) 8(1):e000921. doi: 10.1136/bmjdrc-2019000921

12. Chen Z, Fu S, Wu Z, Chen J, Huang Y, Wang Y, et al. Relationship Between Plasma Angiogenic Growth Factors and Diabetic Foot Ulcers. Clin Chim Acta (2018) 482:95-100. doi: 10.1016/j.cca.2018.03.035

13. Okonkwo UA, Chen L, Ma D, Haywood VA, Barakat M, Urao N, et al. Compromised Angiogenesis and Vascular Integrity in Impaired Diabetic Wound Healing. PloS One (2020) 15:e0231962. doi: 10.1371/journal. pone. 0231962

14. Cianfarani F, Zambruno G, Brogelli L, Sera F, Lacal PM, Pesce M, et al. Placenta Growth Factor in Diabetic Wound Healing: Altered Expression and Therapeutic Potential. Am J Pathol (2006) 169:1167-82. doi: 10.2353/ ajpath.2006.051314

15. Xiao X, Chen Z, Shiota C, Prasadan K, Guo P, El-Gohary Y, et al. No Evidence for Beta Cell Neogenesis in Murine Adult Pancreas. J Clin Invest (2013) 123:2207-17. doi: 10.1172/JCI66323

16. Guo P, Wiersch J, Xiao X, Gittes G. Simplified Purification of AAV and Delivery to the Pancreas by Intraductal Administration. Methods Mol Biol (2019) 1950:373-87. doi: 10.1007/978-1-4939-9139-6_22

17. Xiao X, Fischbach S, Song Z, Gaffar I, Zimmerman R, Wiersch J, et al. Transient Suppression of TGFbeta Receptor Signaling Facilitates Human Islet Transplantation. Endocrinology (2016) 157:1348-56. doi: 10.1210/en.2015-1986

18. Xiao X, Wiersch J, El-Gohary Y, Guo P, Prasadan K, Paredes J, et al. TGFbeta Receptor Signaling Is Essential for Inflammation-Induced But Not Beta-Cell Workload-Induced Beta-Cell Proliferation. Diabetes (2013) 62:1217-26. doi: $10.2337 / \mathrm{db} 12-1428$

19. Guo P, El-Gohary Y, Prasadan K, Shiota C, Xiao X, Wiersch J, et al. Rapid and Simplified Purification of Recombinant Adeno-Associated Virus. J Virol Methods (2012) 183:139-46. doi: 10.1016/j.jviromet.2012.04.004

20. Guo P, Xiao X, El-Gohary Y, Paredes J, Prasadan K, Shiota C, et al. A Simplified Purification Method for AAV Variant by Polyethylene Glycol Aqueous Two-Phase Partitioning. Bioengineered (2012) 4(2):103-6. doi: 10.4161/bioe.22293

21. Gray JT, Zolotukhin S. Design and Construction of Functional AAV Vectors. Methods Mol Biol (2011) 807:25-46. doi: 10.1007/978-1-61779-370-7_2

22. Lang R, Rutschman RL, Greaves DR, Murray PJ. Autocrine Deactivation of Macrophages in Transgenic Mice Constitutively Overexpressing IL-10 Under Control of the Human CD68 Promoter. J Immunol (2002) 168:3402-11. doi: 10.4049/jimmunol.168.7.3402

23. Barrett T, Wilhite SE, Ledoux P, Evangelista C, Kim IF, Tomashevsky M, et al. NCBI GEO: Archive for Functional Genomics Data Sets-Update. Nucleic Acids Res (2013) 41:D991-5. doi: 10.1093/nar/gks1193

24. Li Z, Zhao X, Zhou Y, Liu Y, Zhou Q, Ye H, et al. The Long Non-Coding RNA HOTTIP Promotes Progression and Gemcitabine Resistance by Regulating HOXA13 in Pancreatic Cancer. J Transl Med (2015) 13:84. doi: 10.1186/ s12967-015-0442-z

25. Xiao X, Guo P, Shiota C, Zhang T, Coudriet GM, Fischbach S, et al. Endogenous Reprogramming of Alpha Cells Into Beta Cells, Induced by Viral Gene Therapy, Reverses Autoimmune Diabetes. Cell Stem Cell (2018) 22:78-90.e4. doi: 10.1016/j.stem.2017.11.020

26. Xiao X, Guo P, Prasadan K, Shiota C, Peirish L, Fischbach S, et al. Pancreatic Cell Tracing, Lineage Tagging and Targeted Genetic Manipulations in Multiple Cell Types Using Pancreatic Ductal Infusion of Adeno-Associated Viral Vectors and/or Cell-Tagging Dyes. Nat Protoc (2014) 9:2719-24. doi: 10.1038/nprot.2014.183
27. Xiao X, Gaffar I, Guo P, Wiersch J, Fischbach S, Peirish L, et al. M2 Macrophages Promote Beta-Cell Proliferation by Up-Regulation of SMAD7. Proc Natl Acad Sci USA (2014) 111:E1211-20. doi: 10.1073/pnas.1321347111

28. Wondmkun YT. Obesity, Insulin Resistance, and Type 2 Diabetes: Associations and Therapeutic Implications. Diabetes Metab Syndr Obes (2020) 13:3611-6. doi: 10.2147/DMSO.S275898

29. Okonkwo UA, DiPietro LA. Diabetes and Wound Angiogenesis. Int J Mol Sci (2017) 18(7):1419. doi: 10.3390/ijms18071419

30. Shojaei F, Zhong C, Wu X, Yu L, Ferrara N. Role of Myeloid Cells in Tumor Angiogenesis and Growth. Trends Cell Biol (2008) 18:372-8. doi: 10.1016/ j.tcb.2008.06.003

31. Ferrara N. Vascular Endothelial Growth Factor. Arterioscler Thromb Vasc Biol (2009) 29:789-91. doi: 10.1161/ATVBAHA.108.179663

32. Fischer C, Jonckx B, Mazzone M, Zacchigna S, Loges S, Pattarini L, et al. AntiPlGF Inhibits Growth of VEGF(R)-Inhibitor-Resistant Tumors Without Affecting Healthy Vessels. Cell (2007) 131:463-75. doi: 10.1016/j. cell.2007.08.038

33. Autiero M, Waltenberger J, Communi D, Kranz A, Moons L, Lambrechts D, et al. Role of PlGF in the Intra- and Intermolecular Cross Talk Between the VEGF Receptors Flt1 and Flk1. Nat Med (2003) 9:936-43. doi: 10.1038/nm884

34. Shibuya M. Differential Roles of Vascular Endothelial Growth Factor Receptor-1 and Receptor-2 in Angiogenesis. J Biochem Mol Biol (2006) 39:469-78. doi: 10.5483/BMBRep.2006.39.5.469

35. Eriksson A, Cao R, Pawliuk R, Berg SM, Tsang M, Zhou D, et al. Placenta Growth Factor-1 Antagonizes VEGF-Induced Angiogenesis and Tumor Growth by the Formation of Functionally Inactive PlGF-1/VEGF Heterodimers. Cancer Cell (2002) 1:99-108. doi: 10.1016/S1535-6108(02) 00028-4

36. Incio J, Tam J, Rahbari NN, Suboj P, McManus DT, Chin SM, et al. PlGF/ VEGFR-1 Signaling Promotes Macrophage Polarization and Accelerated Tumor Progression in Obesity. Clin Cancer Res (2016) 22:2993-3004. doi: 10.1158/1078-0432.CCR-15-1839

37. Scatena A, Petruzzi P, Maioli F, Lucaroni F, Ambrosone C, Ventoruzzo G, et al. Autologous Peripheral Blood Mononuclear Cells for Limb Salvage in Diabetic Foot Patients With No-Option Critical Limb Ischemia. J Clin Med (2021) 10(10):2213. doi: 10.3390/jcm10102213

38. Rigato M, Monami M, Fadini GP. Autologous Cell Therapy for Peripheral Arterial Disease: Systematic Review and Meta-Analysis of Randomized, Nonrandomized, and Noncontrolled Studies. Circ Res (2017) 120:1326-40. doi: 10.1161/CIRCRESAHA.116.309045

39. De Angelis B, Gentile P, Orlandi F, Bocchini I, Di Pasquali C, Agovino A, et al. Limb Rescue: A New Autologous-Peripheral Blood Mononuclear Cells Technology in Critical Limb Ischemia and Chronic Ulcers. Tissue Eng Part C Methods (2015) 21:423-35. doi: 10.1089/ten.tec.2014.0245

40. Montanaro M, Meloni M, Anemona L, Giurato L, Scimeca M, Izzo V et al. Macrophage Activation and M2 Polarization in Wound Bed of Diabetic Patients Treated by Dermal/Epidermal Substitute Nevelia. Int $J$ Low Extrem Wounds (2020) 1534734620945559. doi: 10.1177/1534734 620945559

Conflict of Interest: The authors declare that the research was conducted in the absence of any commercial or financial relationships that could be construed as a potential conflict of interest.

Publisher's Note: All claims expressed in this article are solely those of the authors and do not necessarily represent those of their affiliated organizations, or those of the publisher, the editors and the reviewers. Any product that may be evaluated in this article, or claim that may be made by its manufacturer, is not guaranteed or endorsed by the publisher.

Copyright (0) 2021 Zhu, Qian, Jiang, Yang, Duan and Xiao. This is an open-access article distributed under the terms of the Creative Commons Attribution License (CC BY). The use, distribution or reproduction in other forums is permitted, provided the original author(s) and the copyright owner(s) are credited and that the original publication in this journal is cited, in accordance with accepted academic practice. No use, distribution or reproduction is permitted which does not comply with these terms. 